\title{
A Bioassay for Optimization of Macrophage-Conditioned Medium as a Culture Supplement to Promote Hybridoma Cell Survival and Growth
}

\author{
Robert Hnasko, Alice V. Lin, Larry Stanker, and Jeffery McGarvey
}

\begin{abstract}
Macrophage-conditioned medium (MCM) is an important cell culture supplement used to support the survival and growth of newly fused hybridoma cells. The use of macrophage cells, as a part of hybridoma technology, has proven to be an effective and inexpensive source of growth factors that promote the early survival and growth of hybridoma cells. Despite the widespread use of MCM as a hybridoma culture supplement, there is limited guidance and standardization for MCM production to achieve optimal hybridoma support. As an undefined supplement, significant variations in production of MCM may negatively impact hybridoma cell survival and growth. The lack of an available method for standardization of MCM bioactivity has limited validation, optimization, and commercial production. Consequently, variations in batch production of MCM may result in lowquality MCM that limits hybridoma viability and negatively impacts monoclonal antibody production. In this report, we describe a novel bioassay based on the newly generated, MCM-dependent RMH359 hybridoma cell line that can be used to validate MCM bioactivity and standardize production. We demonstrate the utility of the RMH359 bioassay (1) for evaluating MCM hybridoma bioactivity, (2) to define optimal conditions for production of MCM, and (3) as a method for MCM validation and standardization. In conclusion, the RMH359 cell bioassay provides a specific and sensitive assessment of MCM bioactivity in support of hybridoma cell survival and growth.
\end{abstract}

Keywords: bioassay, macrophage, macrophage conditioned medium (MCM), hybridoma, cell culture, supplement

\section{Introduction}

$\mathbf{M}$ ONOCLONAL ANTIBODIES (MAbs) remain an essential molecular tool for the selective and specific detection of biological and chemical antigens. A defining property of an $\mathrm{MAb}$ is its recognition and binding to a single epitope, a characteristic that has been exploited to achieve exquisite antigen selectivity and discrimination. ${ }^{(1)}$ Hybridoma technology has allowed for the production of MAbs to specific antigens following splenocyte cell fusion from an immunized animal with immortalized myeloma-derived cell lines. ${ }^{(2)}$ The resulting hybridoma cells can be screened for selective MAb production and single cells cloned to yield an immortal hybridoma cell line for continuous production of a desired MAb. ${ }^{(3)}$

Hybridoma technology has evolved along with modern cell culture practices, benefiting from refined cell medium formulations and growth supplements, resulting in improved workflow and consistent MAb production. However, there remains a need for cost-effective, animal-derived culture supplements to support early hybridoma cell survival, cloning, and growth. ${ }^{(4,5)}$ Indeed, newly formed hybrid cells often require the addition of a cell feeder layer (i.e., macrophages) or supplementation with a cell-conditioned medium for initial stabilization and growth. ${ }^{(6-8)}$ The use of a cell feeder layer imposes several disadvantages that include interference with hybridoma cell growth and introduction of potential contamination. $^{(9-11)}$ Therefore, cell-conditioned media from continuous cell lines such as fibroblasts ${ }^{(12)}$ or macrophages ${ }^{(7,13,14)}$ have been commonly used as culture medium supplements. Conditioned media collected from the widely available murine macrophage J774A.1 cell line have been shown to improve hybridoma survival and cloning efficiency after cell fusion. ${ }^{(13)}$

Efforts have been made to identify defined medium conditions to support hybridoma growth and increase the efficiency of antibody production. ${ }^{(4,15,16)}$ Proteins such as interlukin-1 (IL-1), tumor necrosis factor, ${ }^{(17)}$ granulocyte-macrophage colony-stimulating factor, ${ }^{(18)}$ and IL- $6^{(16,19)}$ have been shown to support hybridoma growth, resulting in proprietary blends

USDA-ARS, Western Regional Research Center, Albany, California.

(c) Robert Hnasko et al. 2018; Published by Mary Ann Liebert, Inc. This Open Access article is distributed under the terms of the Creative Commons Attribution Noncommercial License (http://creativecommons.org/licenses/by-nc/4.0/) which permits any noncommercial use, distribution, and reproduction in any medium, provided the original author(s) and the source are cited. 
of defined hybridoma growth media. Although these commercial products can be effective, they can be cost prohibitive, and many laboratories rely on in-house production of macrophage-conditioned medium (MCM) to support hybridoma growth during early cell selection and cloning.

Yet, limited guidance and standardization of MCM quality have been reported. This reflects the lack of a defined assay to evaluate the quality of MCM before use as a culture supplement. This study was designed to optimize MCM production by exploiting a new hybridoma cell line RMH359 that is dependent on J774A.1-derived MCM for survival and growth. Using the RMH359 cells, we developed a novel cellular bioassay for evaluation of MCM bioactivity and defined optimal conditions for MCM production to achieve maximal bioactivity in support of hybridoma cell survival and growth. This novel bioassay provides a method for validation and standardization of MCM production for use as a hybridoma culture supplement.

\section{Materials and Methods}

\section{Generation of the RMH359 hybridoma cell line}

Balb/cJ mouse myeloma P3X63AG8 U.1 cells were cultured in advanced RPMI-1640 (Invitrogen, CA) supplemented with $10 \%$ heat-inactivated fetal bovine serum (FBS) and $2 \mathrm{mM}$ Glutamax (Invitrogen) in flasks with $80-\mathrm{cm}^{2}$ culture area (NUNC, DK). Myeloma cells were split 24 hours before fusion with splenocytes from a female Swiss Webster mouse $\left(\mathrm{CFW}^{\circledR}\right.$; Charles Rivers Laboratory, $\left.\mathrm{MA}\right)$ immunized with a hamster brain preparation. Cells were mixed at 1:1 ratio and centrifuged for 10 minutes at $1700 \mathrm{rpm}$. The resulting cell pellet was slowly suspended in $1 \mathrm{~mL}$ of $50 \%$ polyethylene glycol (Sigma-Aldrich, MO), followed by $9 \mathrm{~mL}$ of advanced RPMI-1640. Hybrid cells were initially cultured in medium comprising advanced RPMI-1640 supplemented with $10 \%$ heat-inactivated FBS, $40 \%$ MCM, $0.1 \mathrm{mM}$ sodium hypoxanthine, $0.4 \mu \mathrm{M}$ aminopterin, $0.016 \mathrm{mM}$ thymidine, and $100 \mu \mathrm{g} / \mathrm{mL}$ penicillin/streptomycin for 10 days before feeding with the same growth medium without the addition of aminopterin. Recombinant mouse IL-6 was obtained from R\&D Systems, MN. All cells were maintained at $37^{\circ} \mathrm{C}$ in a humidified $5 \% \mathrm{CO}_{2}$ atmosphere under aseptic conditions. Hybridoma cells were selected by evaluating antibody binding from cell-conditioned media to immobilized hamster brain extract using indirect enzyme-linked immunosorbent assay (ELISA) and cloned $2 \times$ by limiting dilution. Of the surviving hybridoma cell clones, one was designated RMH359, which produced an IgG1 heavy and kappa light chain MAb. Subsequent evaluation of the MAb from the RMH359 culture supernatant showed no specific binding to brain homogenates by either indirect ELISA or Western blot and it is characterized as a nonbinding MAb. Additionally, RMH359 hybridoma cells failed to produce ascites in pristine-primed Balb/cJ mice (Covance, CO; $N=10$ ). The MCM-dependent properties of the RMH359 cell line are stable after cell passage, following multiple rounds of cell division in continuous culture, and upon thaw from liquid nitrogen stock.

\section{Microscopic analysis}

RMH359 cells were seeded at 40,000 cells/well in six-well multidish plates (NUNC, NY, $9.6 \mathrm{~cm}^{2}$ ) in advanced RPMI-
1640 with $10 \%$ FBS medium supplemented with either $50 \%$ $\mathrm{MCM}$ or $50 \%$ nonconditioned macrophage media as controls (see description below). Trypan blue dye exclusion was used to evaluate cell viability by the addition of a Trypan blue solution (Gibco, MA) to $0.1 \%$ for each cell suspension after 8,24 , and 48 hours of cell culture. Phase-contrast digital micrographs were taken with Leica DMI 4000B after incubation of cells with Trypan blue for 3 minutes.

\section{Macrophage cell culture and preparation of MCM}

J774A.1 macrophage cells were seeded at either $1 \times 10^{6}$ or $6 \times 10^{6}$ cells in culture flasks (NUNC, $80 \mathrm{~cm}^{2}$ ) with $15 \mathrm{~mL}$ of advanced RPMI-1640 medium supplemented with $10 \%$ FBS, $5.5 \times 10^{-5} \mathrm{M}$ beta-mercaptoethanol (Invitrogen), $0.5 \mu \mathrm{g} / \mathrm{mL}$ bovine holo-transferrin (Invitrogen), $2 \mathrm{mM}$ Glutamax, and $10 \mathrm{mM}$ HEPES buffer. ${ }^{(13)}$ Culture medium was collected after 24-72 hours of cell growth as MCM, centrifuged at $3000 \mathrm{~g}$ for 5 minutes, filter-sterilized (PES $0.22 \mu \mathrm{m}$ ), stored at $4^{\circ} \mathrm{C}$, and used within 1 week. The same medium composition and conditions were used for the production of control media without the addition of macrophage cells and used at equivalent dilution in RMH359 hybridoma growth media (advanced RPMI +50\% FBS).

\section{MTT assay}

A measurement of cell viability was performed by measuring the reduction of MTT (3-(4,5-dimethylthiazole-2-yl)2,5-diphenyl tetrazolium bromide (Sigma-Aldrich) by viable cells to formazan. ${ }^{(20)}$ The MTT assay performed was similar to that originally described by Mosmann. ${ }^{(21)}$ Briefly, 4000 RMH359 cells were plated in 96-well culture plates (NUNC) and grown for up to 4 days in $200 \mu \mathrm{L}$ of hybridoma growth medium supplemented with $0 \%-50 \% \mathrm{MCM}$ produced under different macrophage culture conditions. MTT was prepared as a filter-sterilized stock solution $(5 \mathrm{mg} / \mathrm{mL}$ in $10 \mathrm{mM}$ phosphate-buffered saline) and added to culture wells at a final concentration of $1 \mathrm{mg} / \mathrm{mL}$ and then incubated at $37^{\circ} \mathrm{C}$ for 4 hours. After the formation of formazan salt, the supernatant was discarded and $100 \mu \mathrm{L}$ of $0.04 \mathrm{~N} \mathrm{HCl}$ in isopropanol was added to dissolve the purple formazan precipitate accumulated in cell mitochondria. The plate was then incubated at room temperature in the dark for 2 hours and the optical density (OD) was measured at a test wavelength of $570 \mathrm{~nm}$ and a reference wavelength of $660 \mathrm{~nm}$ on SpectraMax M5 (Molecular Devices, CA). The OD measurement reported represents the total amount of formazan detected and serves as an indirect measure of viable cell number. Data represent 8-16 replicates per data point and are expressed as mean absorbance units (AU) at $570 \mathrm{~nm} \pm$ SEM.

\section{Caspase-Glo 3/7 assay}

To measure the rate of apoptosis in the absence of MCM, activities of apoptotic markers, caspase- 3 and $-7^{(22)}$, were measured using the caspase-Glo 3/7 assay (Promega, WI). RMH359 cells were plated at two different densities (400 cells and 4000 cells) in 96-well plates and allowed to attach for 24 hours in hybridoma growth medium supplemented with $50 \% \mathrm{MCM}$ before switching to $100 \mu \mathrm{L}$ of medium containing either 50\% MCM or control medium and grown for an additional 7, 24, 48, and 72 hours. A 1:2 dilution of 
caspase 3/7 luminogenic substrate containing the caspase target sequence (DEVD) was added to each well and incubated for 1 hour. Cleavage of the DEVD sequence by caspase- 3 and -7 results in a luminescent signal that is proportional to their cellular activity. ${ }^{(23,24)}$ Luminescence was measured on a Victor X3 plate reader for 0.2 seconds (PerkinElmer, MA) and data represent 12 replicates per time point and are expressed as mean counts per second (CPS) \pm SEM.

\section{CytoTox-Glo ${ }^{\mathrm{TM}}$ cytotoxicity assay}

Cell viability in the absence of MCM was measured by a CytoTox-Glo cytotoxicity assay (Promega). This assay measures cleavage of a membrane-impermeable AAF-Glo ${ }^{\mathrm{TM}}$ substrate by proteases that are released from dead cells. Cells were plated the same way as described for caspase-Glo 3/7 assay and luminescence recorded on a Victor X3 plate reader. After switching to medium containing 50\% MCM or hybridoma control medium, $50 \mu \mathrm{L}$ of AAF-Glo reagent was added directly to cells growing in $100 \mu \mathrm{L}$ of culture medium for 15 minutes at room temperature. The first luminescent signal was recorded (dead cell number), then followed by the addition of the detergent digitonin to lyse remaining viable cells, a second luminescent reading was recorded (total cytotoxicity). The difference between the two luminescent signals is a measure of viable cells per well. The data represent 12 replicates per time point and are expressed as mean CPS \pm SEM.

\section{Results}

We have generated a novel RMH359 hybridoma cell line that remains dependent on MCM for survival and growth. An equivalent number of RMH359 cells were seeded in medium containing $50 \% \mathrm{MCM}$ or macrophage-free control medium and cells were evaluated in the presence of Trypan blue after 8,24 , and 48 hours by phase-contrast microscopy (Fig. 1). Healthy RMH359 hybridoma cells appear round, $\sim 25 \mu \mathrm{m}$ in diameter, and grow as loosely attached clusters that form plaque-like colonies 48 hours after seeding (Fig. 1; left panels). We used a dye exclusion method to evaluate live and
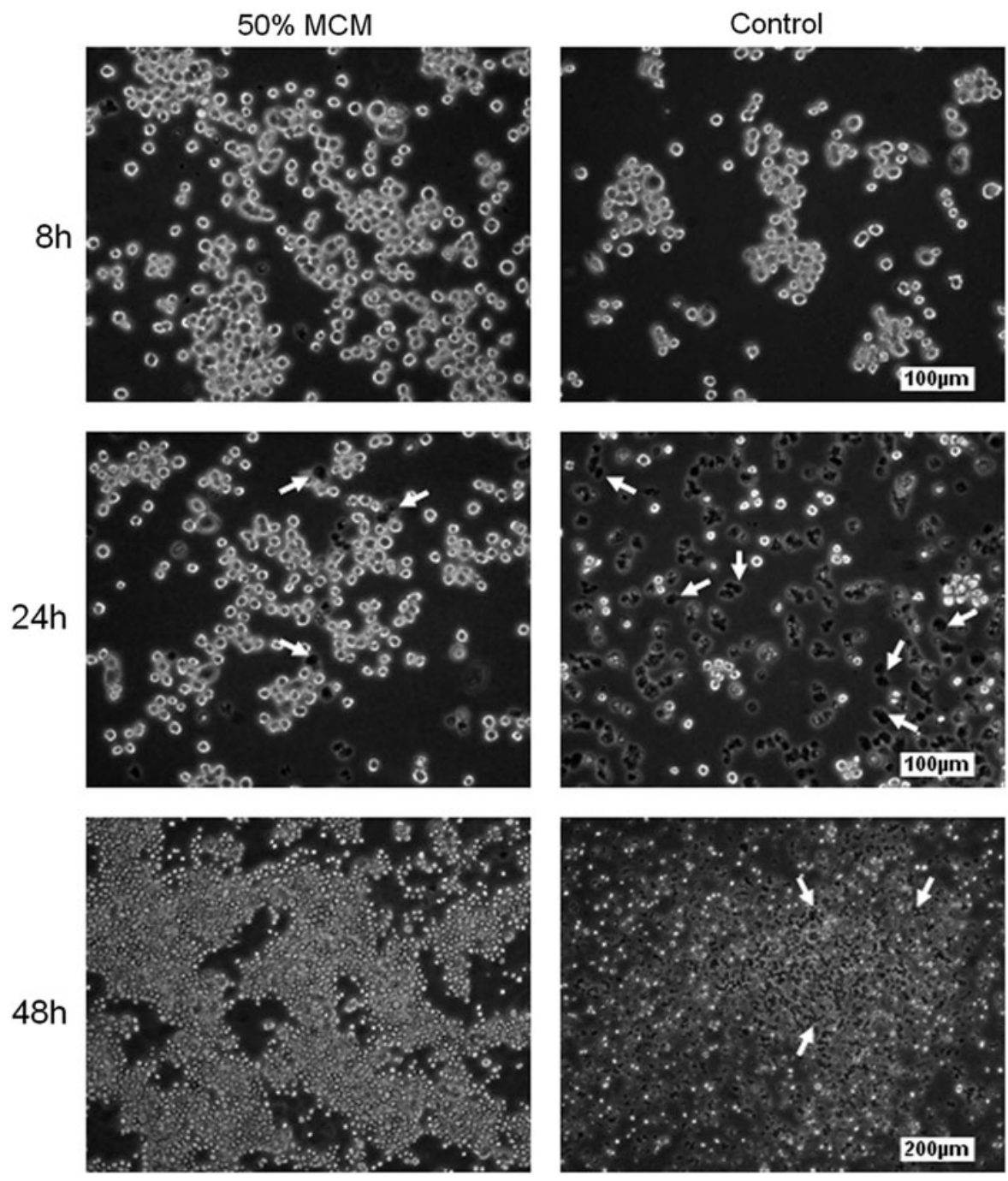

FIG. 1. Phase-contrast photomicrographs comparing Trypan blue exclusion of RMH359 hybridoma cells supplemented with MCM or nonconditioned medium (Control). Micrographs show $0.1 \%$ Trypan blue exclusion of RMH359 cells in hybridoma growth medium supplemented with 50\% MCM (left panels) compared with control cultures (right panels) after 8, 24, and 48 hours. Arrows depict cellular uptake of Trypan blue in some of the nonviable RMH359 cells. MCM, macrophageconditioned medium. 
dead cells in culture by microscopy. Trypan blue is a vital stain that does not cross the intact membranes of living cells, but is selectively absorbed by dead cells that stain blue. ${ }^{(25)} \mathrm{At}$ 8 hours, the majority of cells cultured in either $50 \%$ or control medium were viable with very few dead Trypan blue-stained cells observed (arrows). However, there did appear to be fewer total live cells after 8 hours of growth in the control medium compared with those grown in 50\% MCM. By 24 hours, we observed a dramatic difference in the number of viable RMH359 cells, with most cells grown in 50\% MCM being viable compared with the majority of cells grown in control medium having absorbed the Trypan blue dye. After 48 hours of culture, cells grown in 50\% MCM had proliferated and formed large attached colonies with few Trypan blue-positive cells observed. In contrast, the majority of RMH359 cells cultured in control medium after 48 hours were Trypan blue positive and nonviable.

Dependence of the RMH359 hybridoma cell line on MCM for survival suggested that these cells could be used as a bioassay to determine optimal MCM production from J774A.1. Macrophages were seeded at two different densities in $80-\mathrm{cm}^{2}$ flasks ( 1 or $6 \times 10^{6}$ cells) in $15 \mathrm{~mL}$ of macrophage growth medium for 24 hours to generate $100 \%$ MCM. RMH359 cells were plated in 96-well microplates and cultured in growth medium supplemented with $25 \%$ or $50 \%$ MCM for 4 days and cell viability determined by MTT assay and expressed as \% of MCM-free control (Fig. 2). MCM improved cell viability of RMH359 cells relative to macrophage-free medium control (2-8-fold) and hybridoma growth showed MCM dose dependence, with 25\% MCM half as effective as $50 \%$ MCM in promoting hybridoma growth. Interestingly, increasing the initial macrophage seed density resulted in MCM that was significantly less effective, at either concentration, in promoting RMH359 cell growth $(P<0.05)$. These data suggest that an initial density of $1 \times 10^{6}$

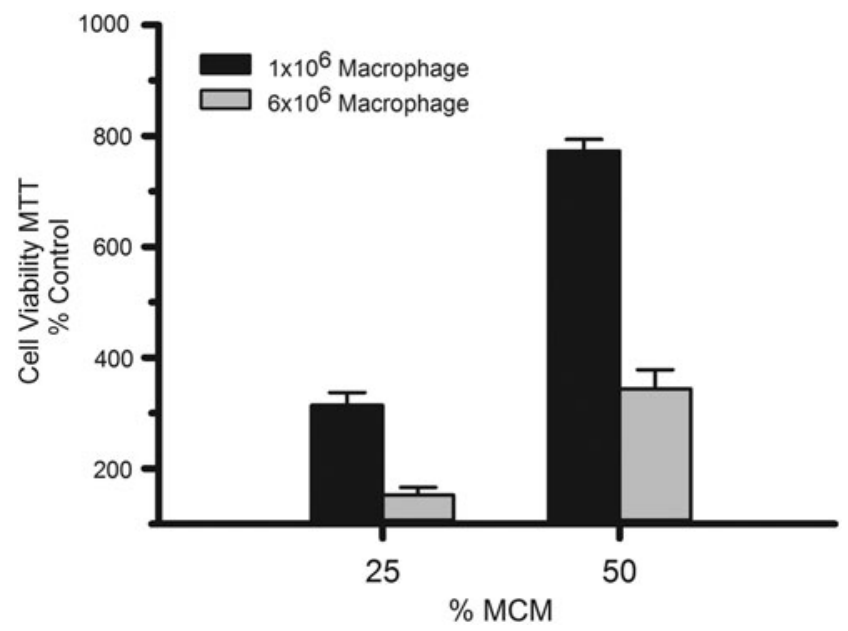

FIG. 2. Impact of macrophage cell density on MCM bioactivity. J744A.1 macrophage cells were seeded in $15 \mathrm{~mL}$ of medium at either $1 \times 10^{6}$ or $6 \times 10^{6}$ cells $/ 80-\mathrm{cm}^{2}$ flasks for 24 hours to produce MCM. MCM was diluted into hybridoma growth medium to either $25 \%$ or $50 \%$ MCM and RMH359 hybridoma cells were incubated for 4 days. The MTT assay (570 nm AU/absorbance) was used to determine cell viability after MCM supplementation and compared with a no MCM control. Data are expressed as percent viable cells compared with control $\pm S E M$. macrophage cells per $80-\mathrm{cm}^{2}$ surface is the optimal density of macrophage cells to yield an effective MCM supplement to support hybridoma cell growth. Maximal RMH359 cell growth (eightfold) was achieved using 50\% MCM generated from $1 \times 10^{6}$ macrophage cells. One-way analysis of variance using the Holm-Sidak all pairwise comparison was performed on MTT $570 \mathrm{~nm}$ absorbance data $(N=8)$ from each group (no MCM control, $25 \%$ and $50 \%$ MCM from $1 \times 10^{6}$ macrophages or $6 \times 10^{6}$ macrophages), with $P<0.05$ considered significant. All comparisons were significant, except $25 \% \mathrm{MCM}$ from $6 \times 10^{6}$ macrophages versus no MCM control and 25\% MCM from $1 \times 10^{6}$ macrophages versus 50\% MCM from $6 \times 10^{6}$ macrophages. Graphical data are expressed as mean $\%$ of no MCM control \pm SEM.

Once we determined the macrophage seeding density and optimal concentration of MCM to achieve maximal RMH359 hybridoma cell growth, we evaluated the impact of macrophage production time on the efficacy of the MCM. Macrophage cells at $1 \times 10^{6}$ were cultured for 24 or 72 hours and the resulting MCM was used to demonstrate the dose-dependent growth of RMH359 with MCM after 4 days by MTT (Fig. 3). At all dilutions tested, 72-hour primed MCM was less effective than 24-hour primed MCM in supporting RMH359 cell viability. Indeed, 25\% 24-hour primed MCM has equivalent bioactivity to 50\% 72-hour primed MCM. However, at $50 \% \mathrm{MCM}$, the difference in cell viability between 24 - and 72-hour primed MCM decreased, suggesting that a 50\% dilution is near maximal for effective hybridoma survival and growth supplementation. Dilutions of MCM above 50\% were less effective in supporting RMH359 cell viability (data not shown). The absence of MCM resulted in nonviable RMH359 cells with MTT absorbance values equivalent to wells with no cells.

RMH359 hybridoma cells supported by 50\% MCM collected after 24 hours did result in statistically higher cell viability $(P<0.05)$ than either 48 - or 72 -hour primed MCM

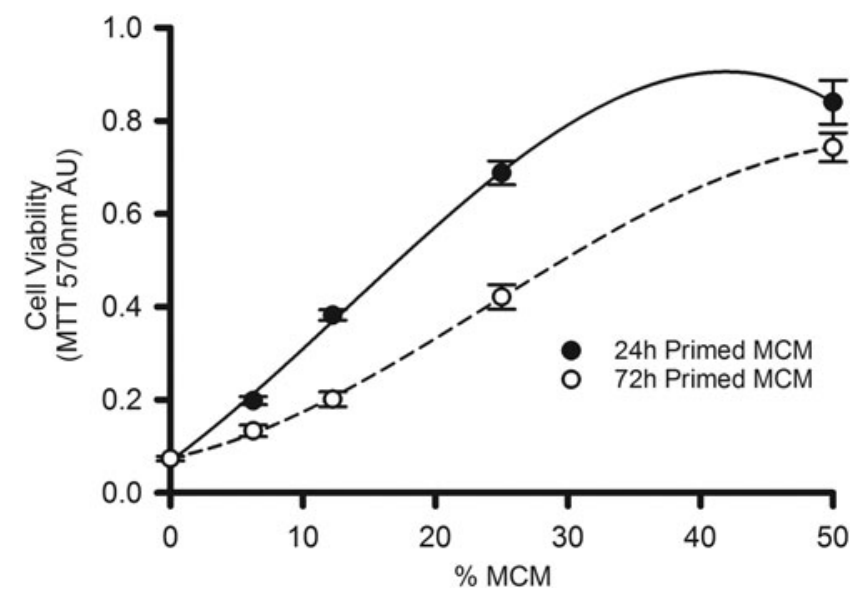

FIG. 3. RMH359 hybridoma cell viability is MCM dose dependent. Macrophages were seeded in $15 \mathrm{~mL}$ of medium at $1 \times 10^{6} \mathrm{cells} / 80-\mathrm{cm}^{2}$ flasks and grown for either 24 hours (๑) or 72 hours $(O)$ to produce MCM. MCM was diluted into hybridoma growth medium from $0 \%$ to $50 \%$ and the MTT assay $(570 \mathrm{~nm}$ AU) was used to determine cell viability after 4 days. The MCM dose-response of viable RMH359 cells was plotted (mean $\mathrm{AU} \pm \mathrm{SEM}$ ) and curve fitting performed using a $3^{\text {rd }}$-order linear regression $\left(r^{2}>0.99\right)$. 


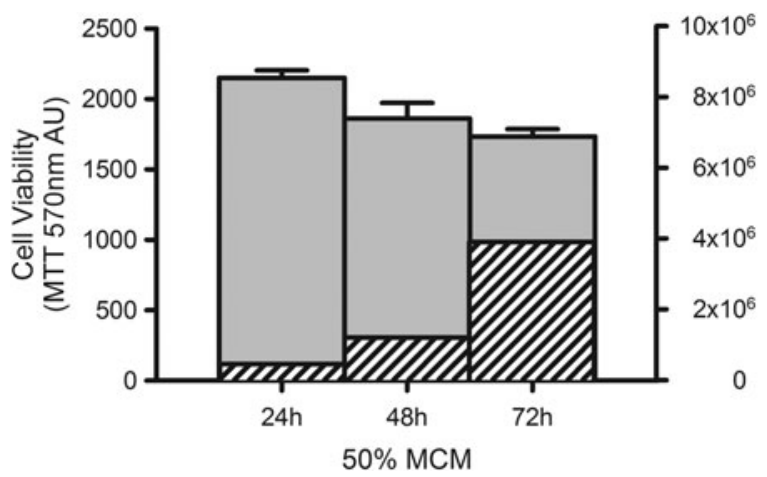

FIG. 4. Improved MCM bioactivity with reduced macrophage cell density and production time. Macrophages were seeded at an initial density of 67,000 cells $/ \mathrm{mL}$ and grown for 24,48 , or 72 hours to produce MCM. The MCM was diluted into hybridoma growth medium to $50 \%$ and the MTT assay (570 nm AU) was used to determine RMH359 cell viability with data expressed as a percent of no MCM control \pm SEM (left axis; solid bars). After MCM harvest, the macrophage cells were collected, counted, and data expressed as cells/ $\mathrm{mL}$ for each time point (right axis; striped bars).

(Fig. 4). We determined the macrophage cell number (cells/ $\mathrm{mL}$ ) at the time of MCM harvest and show a time-dependent increase in macrophage cell number, which is inversely associated with MCM bioactivity as defined by RMH359 cell viability. These data suggest that optimal production of MCM in support of hybridoma cell growth is a subconfluent macrophage density $\left(1 \times 10^{6} / 80 \mathrm{~cm}^{2}\right)$ collected after 24 hours and used in hybridoma culture media at a 40\%-50\% dilution.

The stability of hybridoma growth medium components at $37^{\circ} \mathrm{C}$ in a humidified $5 \% \mathrm{CO}_{2}$ atmosphere over time was evaluated using the RMH359 cell line (Fig. 5). Independent aseptic samples of FBS, MCM, and the complete growth medium (containing advanced RPMI-1640, 10\% FBS, and $40 \% \mathrm{MCM}$ ) were preincubated for 7 and 14 days at $37^{\circ} \mathrm{C}$ in $5 \% \mathrm{CO}_{2}$ without cells. The bioactivity of these preincubated components was then compared with freshly prepared medium made just before use from stock solutions stored at $4{ }^{\circ} \mathrm{C}$ for 4 weeks on RMH359 cell viability after 4 days of growth by MTT. The preincubation of FBS for up to 14 days had no effect on cell viability when used to make complete hybridoma growth media. The stability of MCM was maintained through 7 days of preincubation, but showed a precipitous decline in bioactivity after 14 days of preincubation. Preincubation of complete hybridoma growth medium resulted in a time-dependent decline in bioactivity with $\sim 20 \%$ reduction every 7 days. The difference in the hybridoma support profile between complete hybridoma growth medium and MCM was likely a result of depletion of advanced RPMI1640 components. These data suggest that complete hybridoma growth medium replenishment after $\sim 10$ days of growth would minimize the loss of viable cells as a result of depleted MCM components.

To establish the mechanism of RMH359 cell death, we compared caspase- 3 and -7 activities from cells following MCM withdrawal with those grown in culture medium supplemented with 50\% MCM (Fig. 6A). The withdrawal of the MCM supplement resulted in a significant spike in caspase activity $(P<0.05)$ at 24 hours, followed by a return to levels equivalent to the assay background at 48- and 72-hour time

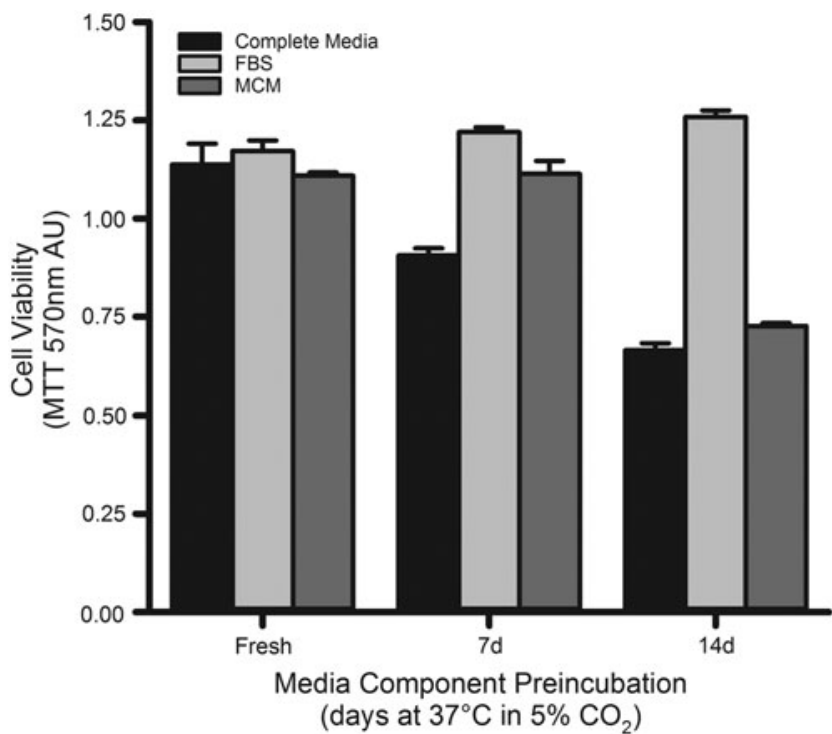

FIG. 5. Stability of MCM with time and temperature. RMH359 cell viability was used to determine the stability of $\mathrm{MCM}$ at $37^{\circ} \mathrm{C}$ in a humidified $5 \% \mathrm{CO}_{2}$ atmosphere. Complete hybridoma growth medium $(10 \% \mathrm{FBS}+50 \% \mathrm{MCM})$ was prepared fresh using MCM and FBS from $4^{\circ} \mathrm{C}$ storage or after their incubation for 7 and 14 days at $37^{\circ} \mathrm{C}$. The incubated FBS and MCM were each used to prepare complete media with other fresh $4^{\circ} \mathrm{C}$ components to $10 \%$ and $50 \%$, respectively. The MTT assay was used to determine RMH359 cell viability after 4 days of growth at $37^{\circ} \mathrm{C}$ and data expressed as mean AU \pm SEM. FBS, fetal bovine serum.

points. By comparison, cells grown with 50\% MCM supplementation showed elevated caspase activity beginning after 48 hours, followed by a time-dependent rise.

Cell viability, as measured by the CytoTox-Glo assay, showed no remaining viable RMH359 cells 48 hours after the withdrawal of MCM (Fig. 6B). By contrast, hybridoma cells seeded at the same density, but maintained in 50\% MCM, showed an increase in viable cell number over time. These data show that with MCM supplementation, the rate of RMH359 cell division is greater than the rate of apoptosis, resulting in an increasing cell number. The increase in apoptosis in MCM-supplemented cultures may reflect resource consumption of cells as they reach a certain density. This may explain the delayed onset of apoptosis observed in MCMsupplemented cultures. MCM likely provides essential factors that promote cell growth resulting in dividing cells that outpace those undergoing apoptosis with a cumulative increase in cell number.

Cytokines play an important role in the survival and growth of immune cells. ${ }^{(26)}$ Several macrophage-secreted factors such as interlukin-6 (IL-6) have been shown to promote hybridoma cell survival and growth in culture. ${ }^{(27)}$ To determine the role of IL-6 as a contributing factor in MCM support of the RMH359 cell line, we performed cell viability experiments by adding IL-6 alone or in combination with suboptimal 12.5\% MCM to hybridoma growth media (Fig. 7). The addition of IL-6 (100 ng/mL) to culture media without MCM failed to promote survival and growth of RMH359 cells and MTT values were equivalent to those obtained without MCM supplementation. However, the addition of 


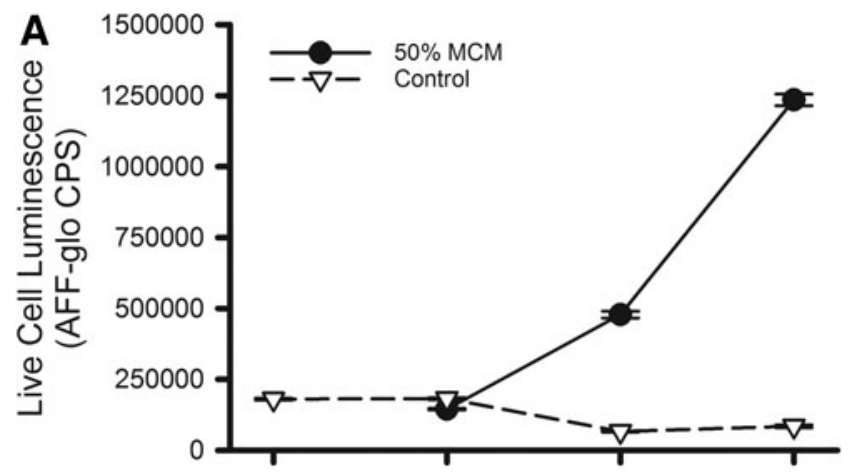

B

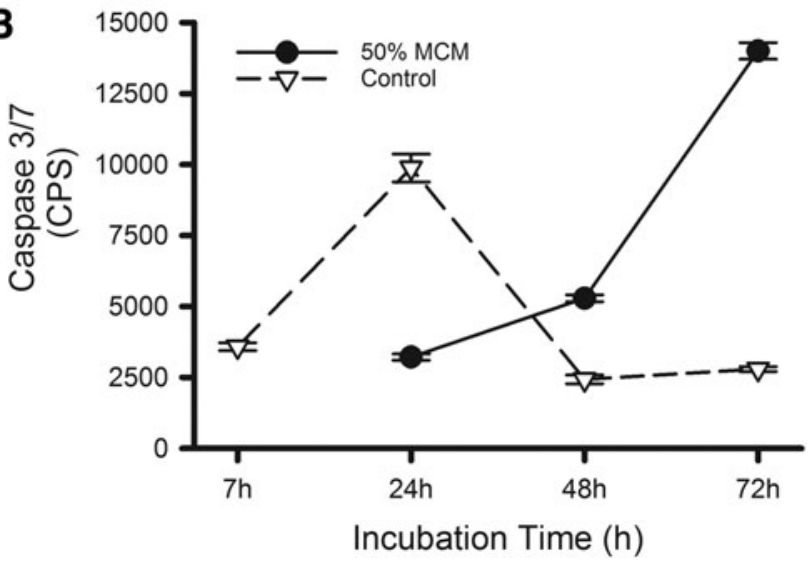

FIG. 6. MCM limits caspase-mediated RMH359 apoptosis. (A) Time-dependent RMH359 cell viability was determined using a CytoTox-Glo ${ }^{\mathrm{TM}}$ luminescent assay in cultures supplemented with 50\% MCM (๑) or following MCM withdrawal $(\nabla)$. (B) In parallel cultures, time-dependent caspase 3/ 7 activity was determined in RMH359 cells supplemented with $50 \%$ MCM (๑) or following MCM withdrawal $(\nabla)$. Luminescent data are expressed as mean CPS \pm SEM. CPS, counts per second.

IL-6 to $12.5 \%$ MCM potentiated the MCM bioactivity resulting in approximately threefold greater cell growth than equivalent MCM alone $(P<0.05)$. We evaluated $12.5 \%$ MCM with IL-6 doses, from 2 to $100 \mathrm{ng} / \mathrm{mL}$, and found that all these concentrations achieved similar cell viability by MTT (data not shown), suggesting that addition of IL-6 at $\leq 2 \mathrm{ng} / \mathrm{mL}$ to MCM would potentiate MCM bioactivity. These data show that addition of IL-6 results in enhanced MCM (eMCM) bioactivity on RMH359 cell growth.

\section{Discussion}

Cell culture supplements such as FBS and MCM provide a rich source of factors necessary for the survival and growth of fragile, newly fused hybrid cells growing under selective pressure from aminopterin. Although most clonal hybridoma cell lines can be weaned from these supplements and grown under defined culture conditions for antibody production, their use during early survival and cell cloning provides beneficial support for cell expansion. It is the goal of cell culture supplementation to promote the postfusion survival and growth of individual hybridomas and thereby increase the selection repertoire of MAb-producing hybridomas as

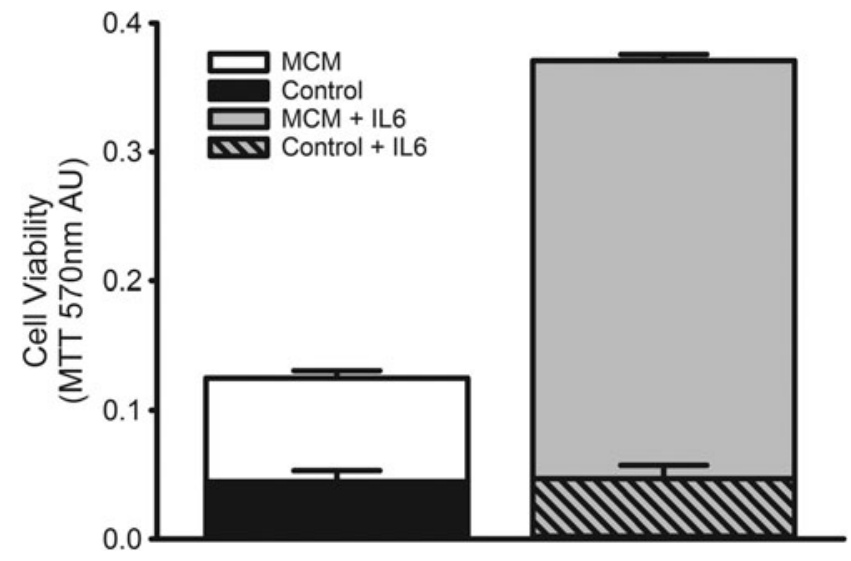

FIG. 7. IL-6 potentiates MCM bioactivity. Control medium alone (black bar) or supplemented with IL-6 $(100 \mathrm{ng} / \mathrm{mL}$; hatched bar) results in nonviable RMH359 cells. Hybridoma growth medium supplemented with $12.5 \%$ MCM (open bar) improves RMH359 cell viability $>2$-fold versus control. The addition of IL-6 (100 ng/mL) along with $12.5 \%$ MCM potentiates MCM bioactivity (gray bar) resulting in $>3$-fold increase in viable RMH359 cells compared with the MCM alone. Cell viability was determined by the MTT assay and data are expressed as mean $570 \mathrm{~nm}$ AU \pm SEM. IL-6, interlukin-6.

well as improve cell cloning efficiencies toward the establishment of a stable cell line.

Although MCM represents a cost-effective and useful product to maximize the efficacy of hybridoma technology, there remains a need for standardization in production and validation to maximize MCM value. In this report, we describe the use of an MCM-dependent RMH359 hybridoma cell line as a bioassay for optimization of MCM production and standardization from J774A.1 macrophages. This represents the first bioassay for validation of MCM bioactivity on hybridoma cell survival and growth, allowing standardization in production and use.

RMH359 cells exhibit MCM dose-dependent growth that is best described by a $3^{\text {rd }}$-order linear regression model ( 24 and 72 hours, $r^{2}=>0.99$ ). These data show that MCM used at $40 \%$ in hybridoma growth medium results in maximal cell growth promotion (Fig. 3). The use of MCM at higher concentrations results in decreased bioactivity and suboptimal hybridoma growth. Importantly, the initial seed density of macrophages (Fig. 2) and their duration of growth (Fig. 3) significantly impact the bioactivity of the MCM product. Higher initial macrophage seed density and increased incubation time are inversely related to MCM bioactivity. The same seed density of macrophages incubated for 72 hours requires more MCM to achieve the same bioactivity as those incubated for 24 hours. MCM likely contains both stimulatory and inhibitory factors that together influence the rate of cell division in a concentration-dependent manner. Consequently, elevated concentrations of MCM (>50\%) likely shift the RMH359 cells toward growth inhibition with a concurrent reduction in their overall growth rate. Accumulation of inhibitory factors or a reduction in stimulatory factors from MCM produced with increased macrophage cell density and incubation duration may also explain the reduced MCM bioactivity under those production conditions. 
The MCM product exhibits excellent retention of bioactivity under storage conditions of $4^{\circ} \mathrm{C}(>1$ month) or from frozen stock. We generally batch produce several liters of $\mathrm{MCM}$ and store aliquots at $-20^{\circ} \mathrm{C}$ for $>1$ year without significant loss in bioactivity (data not shown). However, the MCM product is temperature sensitive $\left(37^{\circ} \mathrm{C} ; 5 \% \mathrm{CO}_{2}\right)$ and gradually loses bioactivity over time (Fig. 5). This reduction is independent of cell presence, with a $\sim 40 \%$ reduction in bioactivity after 14 days at $37^{\circ} \mathrm{C}$. These data suggest that complete medium changes that include MCM should occur for ongoing cell cultures between 7 and 14 days to maximize efficacy of the MCM product.

In the absence of MCM, the RMH359 cell undergoes caspase-mediated apoptosis (Fig. 6). The removal of MCM from RMH359 cells for 17 hours results in a spike in caspase3 and -7 activities (Fig. 6B), followed by a reduction in total live cells (Fig. 6A). These data show that caspase-mediated apoptosis is the mechanism of RMH359 cell death following MCM removal. However, even in the presence of MCM, RMH359 cells undergo some apoptosis (Fig. 6B) with increased caspase- 3 and -7 activities that parallel the rise in live cell number (Fig. 6A). However, the time-dependent increase in total cell number shows that the rate of cell division outpaces that of apoptosis. Therefore, the mitogenic factors in MCM are sufficient to drive cell division and result in cumulative expansion of RMH359 cells.

FBS contains a wide array of mitogenic growth factors that are capable of promoting the survival and growth of cells in culture. ${ }^{(4)}$ Indeed, FBS supplementation of hybridoma culture media is sufficient for generation of hybridoma cell lines. However, the use of MCM likely provides additional factors that facilitate growth and stability of more newly fused, polyploidal hybrid cells. In early hybridoma culture environments, MCM might counteract the effects of accumulating cellular debris from unfused dying cells or help promote growth of low-density hybridomas where autocrine/paracrine growth factor concentrations are insufficient to promote growth from some hybridoma populations. ${ }^{(5)}$ Although the exact composition of factors necessary to best promote MAbproducing hybridoma cell growth in vitro remains undefined, cytokines likely play an important role. ${ }^{(26)}$ IL-6 has been shown to be an important mitogenic factor in B cell hybridoma cell lines. ${ }^{(27-29)}$ In our RMH359 cell bioassay, the addition of IL-6 to media without MCM failed to promote cell survival (Fig. 7). However, IL-6 added to MCM-supplemented medium was synergistic, resulting in enhanced cell growth when compared with the same concentration of MCM without IL-6. These data indicate that the mitogenic activity of MCM on RMH359 cells is not like the result of IL-6 production. Given the synergistic activity of IL- 6 with MCM, we are working toward generation of a stable IL-6-producing J774A.1 macrophage cell line for the production of an eMCM.

The cost, effectiveness, and ease of MCM production support its continued widespread use as a hybridoma cell culture supplement. The RMH359 hybridoma bioassay represents a novel method that provides both validation and optimization of MCM for use in hybridoma culture applications. In this report, optimized MCM production is obtained from $1 \times 10^{6} / 15 \mathrm{~mL} 80 \mathrm{~cm}^{2}$ J774A.1 macrophages grown for 24 hours and used at $40 \%$ in normal hybridoma growth medium containing $10 \%$ FBS. The RMH359 bioassay provides a useful tool for standardization of MCM production that affords a defined specific bioactivity of the product for application in hybridoma technology.

\section{Acknowledgment}

This work was supported and administered under the USDA-ARS National Program in Animal Health (CRIS \#2030-32000-010-00D). The USDA is an equal opportunity provider and employer.

\section{Author Disclosure Statement}

No competing financial interests exist.

\section{References}

1. Wang X, An Z, Luo W, Xia N, and Zhao Q: Molecular and functional analysis of monoclonal antibodies in support of biologics development. Protein Cell 2018;9:74-85.

2. Hnasko RM, and Stanker LH: Hybridoma technology. Methods Mol Biol 2015;1318:15-28.

3. Kohler G, and Milstein C: Continuous cultures of fused cells secreting antibody of predefined specificity. 1975. J Immunol 2005;174:2453-2455.

4. Price PJ: Best practices for media selection for mammalian cells. In Vitro Cell Dev Biol Anim 2017;53:673-681.

5. Spens E, and Haggstrom L: Defined protein-free NS0 myeloma cell cultures: Stimulation of proliferation by conditioned medium factors. Biotechnol Prog 2005;21:87-95.

6. Harlow E, and Lane D: Antibodies: A Laboratory Manual Cold Spring Harbor Lab. Press, Cold Spring Harbor, NY, 1988.

7. Rathjen DA, and Geczy CL: Conditioned medium from macrophage cell lines supports the single-cell growth of hybridomas. Hybridoma 1986;5:255-261.

8. Sugasawara RJ: Hybridoma growth factors. Biotechnology 1988;6:895-902.

9. Andersson J, Coutinho A, Lernhardt W, and Melchers F: Clonal growth and maturation to immunoglobulin secretion in vitro of every growth-inducible B lymphocyte. Cell 1977; 10:27-34.

10. de StGroth SF, and Scheidegger D: Production of monoclonal antibodies: Strategy and tactics. J Immunol Methods 1980;35:1-21.

11. Draber P, Zikan J, and Vogtiskova M: Establishment and characterization of permanent murine hybridomas secreting monoclonal anti-thy-1 antibodies. J Immunogenet 1980;7: 455-474.

12. Walker KZ, Gibson J, Axiak SM, and Prentice RL: Potentiation of hybridoma production by the use of mouse fibroblast conditioned media. J Immunol Methods 1986;88: 75-81.

13. Sugasawara RJ, Cahoon BE, and Karu AE: The influence of murine macrophage-conditioned medium on cloning efficiency, antibody synthesis, and growth rate of hybridomas. J Immunol Methods 1985;79:263-275.

14. Payne WJ, Jr., and Coggin JH, Jr.: Mouse monoclonal antibody to embryonic antigen: Development, cross-reactivity with rodent and human tumors, and preliminary polypeptide characterization. J Natl Cancer Inst 1985;75:527-544.

15. Manna L, Di Febo T, Armillotta G, Luciani M, Ciarelli A, Salini R, and Di Ventura M: Production of monoclonal antibodies in serum-free media. Monoclon Antib Immunodiagn Immunother 2015;34:278-288. 
16. Liu RS, Ta D, Payne J, Coccia M, and Kuus-Reichel K: Generation of murine monoclonal antibodies in serum-free medium. Hybridoma 1998;17:69-72.

17. Le J, Reis LF, and Vilcek J: Tumor necrosis factor and interleukin 1 can act as essential growth factors in a murine plasmacytoma line. Lymphokine Res 1988;7:99-106.

18. Rodrigo A, Valles G, Saldana L, Rodriguez M, Martinez ME, Munuera L, and Vilaboa N: Alumina particles influence the interactions of cocultured osteoblasts and macrophages. J Orthop Res 2006;24:46-54.

19. Aarden L, Lansdorp P, and De Groot E: A growth factor for B cell hybridomas produced by human monocytes. Lymphokines 1985;10:175-185.

20. Berridge MV, Herst PM, and Tan AS: Tetrazolium dyes as tools in cell biology: New insights into their cellular reduction. Biotechnol Annu Rev 2005;11:127-152.

21. Mosmann T: Rapid colorimetric assay for cellular growth and survival: Application to proliferation and cytotoxicity assays. J Immunol Methods 1983;65:55-63.

22. Lakhani SA, Masud A, Kuida K, Porter GA, Jr., Booth CJ, Mehal WZ, Inayat I, and Flavell RA: Caspases 3 and 7: Key mediators of mitochondrial events of apoptosis. Science 2006;311:847-851.

23. Elmore S: Apoptosis: A review of programmed cell death. Toxicol Pathol 2007;35:495-516.

24. Kaufmann SH, Kottke TJ, Martins LM, Henzing AJ, and Earnshaw WC: Analysis of caspase activation during apoptosis. Curr Protoc Cell Biol 2001; Chapter 18:Unit 18.2.
25. Strober W: Trypan blue exclusion test of cell viability. Curr Protoc Immunol 2001;Appendix 3:Appendix 3B.

26. Stenken JA, Poschenrieder AJ: Bioanalytical chemistry of cytokines-A review. Anal Chim Acta 2015;853:95-115.

27. Van Snick J: Interleukin-6: An overview. Annu Rev Immunol 1990;8:253-278.

28. Simpson L, McNiece I, Newberg M, Schetz J, Lynch KR, Quesenberry P, and Isakson PC: Detection and characterization of a B cell stimulatory factor (BSF-TC) derived from a bone marrow stromal cell line. J Immunol 1989;142: 3894-3900.

29. Nordan RP, and Potter M: A macrophage-derived factor required by plasmacytomas for survival and proliferation in vitro. Science 1986;233:566-569.

Address correspondence to: Robert Hnasko USDA-ARS

Western Regional Research Center 800 Buchanan Street Albany CA 94710

E-mail: robert.hnasko@ars.usda.gov

Received: February 2, 2018 Accepted: May 6, 2018 